\title{
Relationship between depressive symptoms and perceived individual level occupational stress among Japanese schoolteachers
}

\author{
Akihiro NAKADA ${ }^{1,2 *}$, Shinichi IWASAKI ${ }^{2}$, Masaru KANCHIKA ${ }^{2}$, Takehisa NAKAO ${ }^{3}$, \\ Yasuhiko DEGUCHI ${ }^{2}$, Akihito KONISHI ${ }^{2}$, Hideyuki ISHIMOTO ${ }^{2}$ and Koki INOUE ${ }^{2}$ \\ ${ }^{1}$ Nara Mental Clinic, Japan \\ ${ }^{2}$ Department of Neuropsychiatry, Osaka City University Graduate School of Medicine, Japan \\ ${ }^{3}$ Nakao Kokoro No Clinic, Japan
}

Received October 10, 2015 and accepted March 14, 2016

Published online in J-STAGE March 25, 2016

\begin{abstract}
Japanese teachers are mentally and physically burdened with various work stressors. This cross-sectional study examined the relationship between depressive symptoms and perceived individual level occupational stress including role problems among Japanese schoolteachers. This study included 1,006 teachers working in public schools in a Japanese city. The Japanese version of Zung's Self-Rating Depression Scale (SDS) was used to evaluate depressive symptoms, and the Generic Job Stress Questionnaire was used to evaluate occupational stress and three measures of social support. Subjects with SDS scores of more than 50 were categorized into the "depressive group." We examined the relationship between depressive symptoms and perceived individual level occupational stress using multiple logistic regression analyses. A total of $202(20.1 \%)$ teachers belonged to the depressive group. We found that high role ambiguity, high role conflict, high quantitative workload, and low social support from family or friends were significantly related to depressive symptoms. To moderate role ambiguity and role conflict experienced by teachers, it is necessary to clarify the priority order of teachers' work. Furthermore, it is necessary to reduce workload by focusing on the content of teachers' work and the setting of education itself. Focusing on these elements will reduce teachers' depressive symptoms.
\end{abstract}

Key words: Teacher, Depressive symptoms, Occupational stress, Generic Job Stress Questionnaire, Role problem

\section{Introduction}

Many studies conducted throughout the world in recent years have highlighted the fact that teaching is a highly stressful occupation. $22 \%$ of German teachers found their occupation extremely stressful ${ }^{1)}$, and the highest proportion of teachers fell into the high stress category ${ }^{2}$. Other

*To whom correspondence should be addressed.

E-mail: twku95975@nike.eonet.ne.jp

(C2016 National Institute of Occupational Safety and Health studies indicate that approximately $70 \%$ of teachers are under frequent stress, with students' discipline problems contributing the most to teacher stress ${ }^{3,4)}$.

In Japan, a nationwide business survey was conducted with Japanese public elementary and junior high school teachers in 2006 $6^{5}$. This survey reported that teachers were distressed mentally and physically due to long working hours, bullying among students, truancy, classroom disruption, parental complications, curriculum revision, teacher evaluation systems, and conflicts among other teachers. Moreover, in Japanese public schools, the incidence of 
teacher sick leave for mental illness has rapidly increased from 2,503 in 2002 to 5,407 in 2011. Two-thirds of teachers' sick leave arises due to mental illness ${ }^{6}$. Therefore, teachers' occupational stress and mental health problems have become pressing issues. There is very little research examining the relationship between these occupational stress in teachers and mental health thus far.

Various occupational stress associates workers' depressive symptoms. High depressive and anxious symptoms in teachers were significantly associated with high job demand and low social support ${ }^{7}$. Chinese university teachers study showed that high effort-reward ratio and overcommitment tendency were risk factors for depressive symptoms, whereas psychological capital might be protective against depressive symptoms ${ }^{8)}$.

Additionally, teachers' work is composed of a multitude of often competing responsibilities, particularly in Japan. As mentioned above, Japanese teachers have various duties to their students besides education, such as their essential educational work, office work, participation in training and research activities, Parent Teacher Association (PTA) activities, and community activities. An international, large-scale survey, which is known as the OECD Teaching and Learning International Survey (TALIS 2013), has been conducted to focuses on the working conditions of teachers and the learning environment in 34 countries in $2013^{9)}$. This survey shows that teachers in Japan report spending only 18 hours teaching in a week, meaning they spend substantially more time on other tasks related to their job than they spend actually teaching. It is supposed that teachers experience their role as "teacher" confusing. Some previous studies have focused on the role problems of teachers, particularly the association of role conflict and role ambiguity with burnout. Schwab found that role conflict and role ambiguity explained a significant amount of the variance in feelings of emotional exhaustion and negative attitudes toward students ${ }^{10)}$, and Jackson found that emotional exhaustion was strongly associated with role conflict ${ }^{11)}$. Therefore, role problems, including "role conflicts" (e.g., being required to do things that they do not perceive to be part of their job) and "role ambiguity" (e.g., a situation when an employee does not have adequate information to perform a task or does not fully understand the requirements of the task) were supposed to intensify teachers' levels of occupational stress. Inoue found that job control and role ambiguity can be important predictors of longterm sick leave owing to depressive disorders among male employees in manufacturing factories ${ }^{12)}$. As mentioned above, teachers have unique role problems, and teachers' role problem is quite different from blue-collar workers' one. However, the role problems have received little attention in research literature, despite the fact that these factors are also important work stressors for teachers.

Therefore, we hypothesized that perceived individual level occupational stress and social support based on the DC/S model, added role problems are associated with depressive symptoms among teachers. This study aimed to clarify the relationship between perceived individual level occupational stress, role problems and depressive symptoms among teachers.

\section{Subjects and Methods}

\section{Participants}

This study was based on data from a cross-sectional study of public school teachers in a city in the Kansai region of Japan in 2013. We mailed anonymous questionnaires with a return-mail envelope to participants, including teachers, principals, and vice-principals, who were attached to kindergarten schools, primary schools, junior high schools, high schools, and special schools in the city. To protects individual privacy, we did not obtain the identifier of school which each subjects belongs to. In addition, to obtain informed consent, a letter was enclosed describing the aims and procedures of the study, particularly to assure participants that the survey was anonymous and voluntary and that no individual would be identified in analyzing or reporting the data.

\section{Ethics statement}

The study protocol was approved by the Human Subjects Review Committee of Osaka City University (Approved \#1409). The data obtained includes only anonymized participant data.

\section{Demographic and occupational variables}

The demographic variables included age, sex, and marital status (currently married, unmarried, divorced, and widowed). Occupational variables included the types of school the respondents worked at (kindergarten, primary school, junior high school, high school, or special needs education school) and overtime hours per month (under 46, between 46 and 80 , and over 80 ).

\section{Generic Job Stress Questionnaire (GJSQ)}

We measured perceived individual level occupational stress using the Japanese version of the Generic Job Stress Questionnaire (GJSQ) ${ }^{13)}$. The GJSQ is a useful question- 
naire for assessing various aspects of occupational stress, including occupational stress and stress reactions at the group and individual level. The GJSQ has been shown to have sufficient reliability and validity. The original authors of the GJSQ permit the use of the measure's independent subscales for occupational stress ${ }^{14}$. We chose four subscales to assess perceived individual level occupational stress (job control, quantitative workload, role conflict, and role ambiguity) and three measures of social support (from supervisors, from co-workers, and from family or friends) according to the $\mathrm{DC} / \mathrm{S}$ model for the assessment of perceived individual level occupational stress. The quantitative workload is a four-item scale that measures the amount of work a person has to deal with on a daily basis. Job control is a 16-item scale that assesses the degree to which the individual feels that his or her tasks, workplace settings, and decisions at work are controllable. Role conflict is an eight-item scale that measures how often workers experience role conflict with each other. Role ambiguity is a six-item scale that measures how clearly the worker understands what is expected of him or her for adequately performing a role or task. The amount of social support the respondents received from supervisors, co-workers, and family or friends was measured on a four-item scale. The item descriptions of quantitative workload, role conflict, and role ambiguity are negatively oriented, such that higher scores indicate greater stress. In contrast, the item descriptions of job control and social support are positively oriented, so that higher scores indicate lower stress.

\section{Zung's Self-Rating Depression Scale (SDS)}

Depressive symptoms were evaluated using the Japanese version of Zung's Self-Rating Depression Scale (SDS) ${ }^{15}$, which has been used in previous studies and clinical settings in Japan. The scores for the 20 items were added up, and the total score was converted to a $20-80$ point scale. A higher total score indicates more severe depressive symptoms. Previous studies have demonstrated the reliability and validity of the SDS in both Japan and Western countries ${ }^{16-19}$. Based on previous studies, we defined the morbidity cut-off point on the SDS as $50^{20,21)}$. Individuals with SDS scores of more than 50 were categorized as the "depressive group" and displayed moderate or severe depressive symptoms, and those with SDS scores of less than 49 were categorized as the "non-depressive group."

\section{Statistical analysis}

Logistic regression analyses were used to estimate the odds ratio (ORs) for belonging to the depressive group based on seven GJSQ subscales (job control, quantitative workload, role conflict, role ambiguity, social support from supervisors, social support from co-workers, and social support from family or friends) subdivided into low, middle, or high categories according to the tertile scores. A multivariate model was subsequently used to estimate the ORs for belonging to the depressive group, adjusted for demographic variables (sex, age, and marital status) and occupational variables (type of school and overtime work) and the seven subdivided GJSQ subscales. The multilevel analysis (school level) was not performed, because we could not obtain the identifier of school which each teachers belongs to. All statistical analyses were performed using the Statistical Package for the Social Sciences version 22.0 (IBM Software Group, Chicago, IL).

\section{Results}

We mailed anonymous questionnaires with a return-mail envelope to 2,876 participants, including teachers, principals, and vice-principals, who were attached to 60 kindergarten schools, 299 primary schools, 130 junior high schools, 21 high schools, and 11 special schools in the city. A total of 1,912 individuals who agreed with the aims of this study returned the questionnaire in the sealed envelope, providing a response rate of $66.5 \%$. After excluding teachers who had at least one missing entry in the questionnaire, the final number of respondents included in the analyses was 1,545 . In this study, we used only teacher data $(N=1,006$; excluding principals and vice-principals) to avoid the effects of position.

Table 1 shows the characteristics of 1,006 subjects. The average age ( \pm standard deviation: $S D$ ) of subjects was $39.7 \pm 11.6$ years old. A total of 600 subjects $(59.6 \%)$ were women and 406 (40.4\%) were men. A total of 553 subjects $(55.0 \%)$ were married. The highest proportion worked at a junior high school (33.8\%).

Results of the SDS and GJSQ subscales are shown in Table 2. The mean score and SD of the SDS of all subjects was $41.6 \pm 9.7$. In this study, $202(20.1 \%)$ were classified into the depressive group, with SDS points of more than 50 , and $804(79.9 \%)$ were classified into the non-depressive group. The mean scores and SD for each group were $56.2 \pm 5.5$ points and $38.0 \pm 6.7$.

Table 3 shows the result of multivariate logistic regression analysis using each GJSQ factor and individual factors as independent variables and the depressive group as a dependent variable, and calculated their ORs. It was found that the higher subdivided stress group by "quan- 
Table 1. Subject's characteristics

\begin{tabular}{llrrr}
\hline & & $\mathrm{N}$ & $(\%)$ & Mean \pm SD \\
\hline Age & & 1,006 & & $39.7 \pm 11.6$ \\
Sex & Female & 600 & $(59.6 \%)$ & \\
& Male & 406 & $(40.4 \%)$ & \\
Marital status & 553 & $(55.0 \%)$ & \\
& Married & 395 & $(39.3 \%)$ & \\
& Unmarried & 58 & $(5.7 \%)$ & \\
& Divorced, Widowed & 146 & $(14.5 \%)$ & \\
School category & Kindergarten & 315 & $(31.3 \%)$ & \\
& Primary school & 340 & $(33.8 \%)$ & \\
& Junior high school & 98 & $(9.7 \%)$ & \\
& High school & & $(10.6 \%)$ & \\
& Special needs edu- & 107 & \\
& cation school & 640 & $(63.6 \%)$ \\
Overtime hours & $<45$ & 249 & $(24.8 \%)$ & \\
& $46 \leq<80$ & 117 & $(11.6 \%)$ & \\
& $81 \leq$ & & \\
\hline
\end{tabular}

Table 2. SDS and GJSQ scores

\begin{tabular}{llc}
\hline & Mean \pm SD & N (\%) \\
\hline SDS score & \multicolumn{3}{c}{} \\
$\quad$ Total & $41.6 \pm 9.7$ & 1,006 \\
$\quad$ Non-depressive group $(<50)$ & $38.0 \pm 6.7$ & $804(79.9 \%)$ \\
$\quad$ Depressive group ( $\geq 50)$ & $56.2 \pm 5.5$ & $202(20.1 \%)$ \\
GJSQ scores (range) & & \\
$\quad$ Quantitative Workload (17-55) & $43.0 \pm 7.1$ & \\
Job Control (16-80) & $38.6 \pm 11.4$ & \\
Role Conflict (8-56) & $34.2 \pm 7.4$ & \\
Role Ambiguity (6-42) & $23.5 \pm 5.6$ & \\
$\quad$ Social Support from supervisor (4-20) & $13.0 \pm 3.5$ & \\
$\quad$ Social Support from coworker (4-20) & $14.6 \pm 3.3$ & \\
Social Support from family/friends (4-20) & $15.3 \pm 3.4$ & \\
\hline
\end{tabular}

SDS: Zung's Self-Rating Depression Scale. GJSQ: Generic Job Stress Questionnaire.

Higher score of each factors means higher stress except job control and social support. Higher job control and social support score means lower stress.

titative workload" ( $\mathrm{OR}=2.89$, adjusted), "role conflict" $(\mathrm{OR}=3.81$, adjusted), "role ambiguity" $(\mathrm{OR}=3.12$, adjusted), and "social support from family or friends" $(\mathrm{OR}=2.20$, adjusted) was associated with the presence of depressive symptoms using both a crude model and an adjusted model (adjusted for age, gender, marital status, school category, and overtime work). Additionally, the middle score group for "role conflict" was associated with the presence of depressive symptoms. "Age" was a demographic variable associated weakly with depressive symptoms $(\mathrm{OR}=0.98)$.

\section{Discussion}

In this study, out of 1,006 subjects, 202 (20.1\%) teachers were evaluated as having depressive symptoms. Furthermore, we examined the relationships between perceived individual level occupational stress and depressive symptoms. Using multivariate logistic regression analysis, the two high role problems of teachers (high role conflict and ambiguity) as job stressors, high quantitative workload as job demand, low social support from family or friends, and age as an individual factor related to depressive symptoms. Job control, social support from supervisors and coworkers, and other individual factors were not related to having depressive symptoms. This is the first study demonstrated the relationship between role stressors and depressive symptoms among teachers.

The study evaluated the presence of depressive symptoms in healthy people using SDS and reported that $12.7 \%$ of subjects had 50 or more points as a depressive group ${ }^{21}$. The measurement of SDS scores in a general population were conducted in Iwate Prefecture, Japan ${ }^{22}$. It showed that the mean SDS score for all subjects $(\mathrm{n}=5,547)$ was 39.3 points high or moderate SDS scores $(\geq 48)$ were found in $13.7 \%$ of the subjects. In this study, we regarded 50 or more points on the SDS score as indicating depressive symptoms. This means that $20.1 \%$ of subjects fit this category. The study reported that $36-38 \%$ of teachers had depressive symptoms, measured by 50 or more points on the SDS score ${ }^{23)}$. Our result shows that the prevalence of depressive symptoms in teachers was lower than that in these studies but higher than that of healthy people.

Some previous studies have reported on the workload of teachers. In 2005, the Institute for Science of Labour conducted a survey on 6,000 Japanese teachers' health and reported that teachers' average overtime working hours amounted to 53.1 hours a month ${ }^{24)}$. This is far more overtime than that undertaken by full-time employees in companies, who work on average 10.6 hours of overtime a month in $\operatorname{Japan}^{25)}$. Across countries, teachers reported spending an average of 38 total hours working in a week, whereas Japanese teachers reported a total of 54 hours that was the longest in the 34 countries participating in TALIS $2013^{9)}$. No relationship was observed between overtime work and depressive symptoms in this study, but quantitative workload was significantly related to depressive symptoms. Working overtime is common for Japanese teachers. Therefore, it is supposed that overtime work does not mean high quantitative workload, and the workload they feel is more important for depressive symptoms than 
Table 3. Analysis of risk factors for depressive symptoms by multivariate logistic regression analysis

\begin{tabular}{|c|c|c|c|c|c|c|c|}
\hline \multirow{2}{*}{ Occupational stress and social supports } & & \multicolumn{3}{|c|}{ Crude model } & \multicolumn{3}{|c|}{ Adjusted model $\dagger$} \\
\hline & & $\mathrm{OR}$ & $(95 \% \mathrm{CI})$ & $p$ & OR & $(95 \% \mathrm{CI})$ & $p$ \\
\hline \multirow[t]{3}{*}{ Quantitative Workload } & (Low) & 1.00 & & & 1.00 & & \\
\hline & Middle & 1.45 & $(0.97-2.17)$ & & 1.26 & $(0.79-1.99)$ & \\
\hline & High & 3.81 & $(2.60-5.59)$ & $* *$ & 2.89 & $(1.76-4.73)$ & $* *$ \\
\hline \multirow[t]{3}{*}{ Job Control } & (High) & 1.00 & & & 1.00 & & \\
\hline & Middle & 1.34 & $(0.83-2.15)$ & & 0.83 & $(0.48-1.45)$ & \\
\hline & Low & 2.85 & $(1.83-4.45)$ & $* *$ & 1.07 & $(0.62-1.83)$ & \\
\hline \multirow[t]{3}{*}{ Role Conflict } & (Low) & 1.00 & & & 1.00 & & \\
\hline & Middle & 2.99 & $(1.89-4.71)$ & $* *$ & 1.70 & $(1.02-2.82)$ & * \\
\hline & High & 9.27 & $(5.86-14.66)$ & $* *$ & 3.81 & $(2.23-6.51)$ & $* *$ \\
\hline \multirow[t]{3}{*}{ Role Ambiguity } & (Low) & 1.00 & & & 1.00 & & \\
\hline & Middle & 1.95 & $(1.18-3.20)$ & $* *$ & 1.58 & $(0.91-2.75)$ & \\
\hline & High & 6.10 & $(3.89-9.57)$ & $* *$ & 3.12 & $(1.80-5.40)$ & $* *$ \\
\hline \multirow[t]{3}{*}{ Social Support from supervisor } & (High) & 1.00 & & & 1.00 & & \\
\hline & Middle & 1.79 & $(1.16-2.78)$ & $* *$ & 1.23 & $(0.73-2.08)$ & \\
\hline & Low & 4.55 & $(2.96-7.01)$ & $* *$ & 1.73 & $(0.99-3.04)$ & \\
\hline \multirow[t]{3}{*}{ Social Support from coworker } & (High) & 1.00 & & & 1.00 & & \\
\hline & Middle & 2.03 & $(1.33-3.11)$ & $* *$ & 1.40 & $(0.85-2.31)$ & \\
\hline & Low & 3.91 & $(2.56-5.97)$ & $* *$ & 1.67 & $(0.97-2.90)$ & \\
\hline \multirow[t]{3}{*}{ Social Support from family/friends } & (High) & 1.00 & & & 1.00 & & \\
\hline & Middle & 1.06 & $(0.69-1.62)$ & & 0.87 & $(0.53-1.43)$ & \\
\hline & Low & 2.90 & $(1.96-4.28)$ & $* *$ & 2.20 & $(1.35-3.58)$ & $* *$ \\
\hline Age & & & & & 0.98 & $(0.96-1.00)$ & $*$ \\
\hline \multirow[t]{2}{*}{ Sex } & (Female & & & & 1.00 & & \\
\hline & Male & & & & 0.89 & $(0.58-1.38)$ & \\
\hline \multirow[t]{3}{*}{ Marital status } & (Marrie & & & & 1.00 & & \\
\hline & Unmarr & & & & 0.76 & $(0.44-1.11)$ & \\
\hline & Divorce & d, Wido & owed & & 2.12 & $(0.74-2.32)$ & \\
\hline \multirow[t]{5}{*}{ School category } & (Kinder & sarten) & & & 1.00 & & \\
\hline & Primary & school & & & 0.68 & $(0.37-1.25)$ & \\
\hline & Junior $\mathrm{h}$ & igh sch & & & 0.67 & $(0.35-1.26)$ & \\
\hline & High scl & nool & & & 0.64 & $(0.28-1.47)$ & \\
\hline & Special & needs e & ducation school & & 1.53 & $(0.73-3.17)$ & \\
\hline \multirow[t]{3}{*}{ Overtime hours } & $(<45)$ & & & & 1.00 & & \\
\hline & $46 \leq<8$ & & & & 0.70 & $(0.48-1.20)$ & \\
\hline & $81 \leq$ & & & & 1.31 & $(0.99-4.52)$ & \\
\hline
\end{tabular}

OR: Odds Ratio. CI: Confidence Interval. ${ }^{*} p<0.05$ compared with Not depressed group. ${ }^{* *} p<0.01$ compared with Not depressed group.

Parenthesis denotes reference category.

$\dagger$ : Adjusted for all listed variables.

actual overtime work.

In general, teachers' job control is considered to be higher than that of other occupations ${ }^{26)}$ because teachers have a more subjective judgmental sense and feel more able to decide on the pace, procedure, and policy of their work. As this study shows, there is no relationship between teachers' job control and depressive symptoms via logistic regression analysis. The association between job control and occupational stress is controversial. We have reported that lower job control reduced ORs of those belonging to the high verbal aggression group among Japanese teach$\mathrm{ers}^{27}$. Studies on occupational stress and burnout have found that excess job control induces the requirement of more responsibility and excess workload, and this has resulted in physical and psychological exhaustion among Japanese teachers ${ }^{26)}$. In this study, the middle job control group showed the lowest ORs, and high and low job control groups showed higher (although not significantly higher) ORs than the middle job control group. This may suggest that having insufficient or excess job control 
relates to depressive symptoms; however, among Japanese teachers, having moderate job control does not.

Some studies reported that teachers' work is difficult to define clearly and have indicated that the ambiguity of unlimited standards for achievement is a factor promoting teachers' job stress ${ }^{28}$. In addition, the lack of a time framework is considered to be relevant. For example, teachers have to work at home to prepare the next day's teaching materials or go to work for extracurricular activities on a holiday. TALIS 2013 reported that extracurricular activities were an important aspect of teachers' work in Japan, where teachers report spending eight hours in a week on extracurricular activities, far above the TALIS average of two hours ${ }^{9}$. Working hours excepting teaching undertaken by Japanese teachers (e.g., individual planning on preparation of lessons, team work and dialog with colleagues, student counseling, participation in school management, and general administrative work) tended to be longer than those in other countries. These are exemplary of the characteristic role conflict experienced by teachers. Several studies have been conducted on the relationship between role conflict and burnout, reporting that teachers' role conflict affects emotional robustness ${ }^{29}$. This study also showed significant relationships between role conflict and depressive symptoms in teachers.

Our findings show that teachers' sense of role ambiguity related to depressive symptoms in this study. Teachers are often asked about the role of being a lecturer in cram or prep school in addition to the essential role of being an educator from students' parents. This is an example of role ambiguity. They also have to perform tasks, such as office work, community activities, among others, besides essential work in education. Thus, the role played by teachers is unclear. Preventing depressive symptoms is necessary for reducing the role problems experienced by teachers.

In this study, only social support from family or friends significantly reduced depressive symptoms. There are very few supervisors (i.e., principal and some vice-principals) who should manage and support teachers. As mentioned above, coworkers (teachers) also have to perform several tasks to have enough time to support each other. These situations may reconcile the requirement for social support from associates in the workplace, and in order to socialize with people (e.g., family or friends) off the job, it is essential for them to feel at ease. To be supported socially by family or friends, reducing job demands such as through overtime hours and job clarification is required.

This study has some limitations. First, we only evaluated the relationship between perceived individual level occupational stress and depressive symptoms. The DC/S model is judged not only by depressive symptoms but also by the presence of physiological changes. Further studies using each rating scale for other psychological symptoms except depression should also be conducted. Furthermore, fitness regarding each teacher's perceived individual level occupational stress relating to the DC/S model should be studied further. Second, in this study, we used a self-rating questionnaire. The scores on a self-rated depression scale were not always identical with a clinical diagnosis of depression ${ }^{30)}$. Although the practice of individual interviews in a large study is difficult, examination including individual interviews by psychiatrists is expected in a future study. Third, a cross-sectional method of research was used in this study. Further review of these items is needed to track subjective depressive symptoms using longitudinal methods and to examine the relationship between depression and changes in occupational stress. Fourth, the data were obtained from one city in Japan. Therefore, it may be difficult to generalize the findings to other regions and cultures. Fifth, we could not perform the multilevel analysis (school level) because of lacking in the identifier of school. Therefore we could analyze and discuss only about individual level stress. Further studies are needed in order to clarify the effect of school level stress.

In sum, teachers' high quantitative workload, high levels of role conflict and role ambiguity, and low social support from family or friends are stressors that relate to depressive symptoms. Modulating role ambiguity and role conflict experienced by teachers by clarifying the order of priority of teachers' work, reducing workload by focusing on the contents of teachers' work, and setting education itself as essential are modifications that are required to reduce teachers' depressive symptoms.

\section{References}

1) Unterbrink T, Hack A, Pfeifer R, Buhl-Grießhaber V, Müller U, Wesche H, Frommhold M, Scheuch K, Seibt R, Wirsching M, Bauer J (2007) Burnout and effort-rewardimbalance in a sample of 949 German teachers. Int Arch Occup Environ Health 80, 433-41.

2) Smith A, Brice C, Collins A, McNamara R, Matthews V (2000) The scale of occupational stress: A further analysis of the impact of demographic factors and type of job. Health and Safety Executive Books, Sadbury.

3) Burke RJ, Greenglass ER, Schwarzer R (1996) Predicting teacher burnout over time: Effects of work stress, social support, and self-doubts on burnout and its consequences. Anxiety Stress Coping 9, 261-75.

4) Rudow B (1999) Stress and Burnout in the Teaching Profes- 
sion: European Studies, Issues, and Research Perspectives Understanding and Preventing Teacher Burnout. Cambridge University Press, Cambridge.

5) Tsuchiya N, Nishikido N (2007) Trends in research on mental health situation and measures of teacher. Occupational mental health 15, 271-5 (in Japanese).

6) Ministry of Education, Culture, Sports, Science and Technology in Japan. STATISTICAL ABSTRACT 2012 edition 1.1 School Education. http://www.mext.go.jp/english/ statistics/1302870.htm. Accessed Dec 29, 2015.

7) Borrelli I, Benevene P, Fiorilli C, D’Amelio F, Pozzi G (2014) Working conditions and mental health in teachers: a preliminary study. Occup Med (Lond) 64, 530-2.

8) Gong Y, Han T, Chen W, Dib HH, Yang G, Zhuang R, Chen Y, Tong X, Yin X, Lu Z (2014) Prevalence of anxiety and depressive symptoms and related risk factors among physicians in China: a cross-sectional study. PLoS One 9, e103242.

9) Organisation for Economic Co-operation and Development (OECD) (2014) TALIS 2013 Results: An International Perspective on Teaching and Learning. OECD publishing, Paris.

10) Schwab RL, Iwanicki EF (1982) Perceived Role Conflict, Role Ambiguity, and Teacher Burnout. Educ Adm Q 18, 60-74.

11) Jackson SE, Schwab RL, Schuler RS (1986) Toward an understanding of the burnout phenomenon. J Appl Psychol 71, 630-40.

12) Inoue $A$, Kawakami $N$, Haratani $T$, Kobayashi $F$, Ishizaki M, Hayashi T, Fujita O, Aizawa Y, Miyazaki S, Hiro H, Masumoto T, Hashimoto S, Araki S (2010) Job stressors and long-term sick leave due to depressive disorders among Japanese male employees: findings from the Japan Work Stress and Health Cohort study. J Epidemiol Community Health 64, 229-35.

13) Haratani $T$ (1993) Reliability and validity of the Japanese version of NIOSH Generic Job Questionnaire. Japanese Journal of Industrial Health 35, 214-5 (in Japanese).

14) Hurrell JJ Jr, McLaney MA (1988) Exposure to job stress--a new psychometric instrument. Scand J Work Environ Health 14 Suppl 1, 27-8.

15) Zung WW (1965) A Self-Rating Depression Scale. Arch Gen Psychiatry 12, 63-70.

16) Buss AH, Perry M (1992) The aggression questionnaire. J Pers Soc Psychol 63, 452-9.

17) Deguchi $Y$, Inoue $K$, Muramatsu $T$, Iwasaki S, Yamauchi $T$, Nakao T, Muroya M, Kobayashi Y, Kato Y, Kiriike N (2013) Relationships between occupational stress and depressive symptoms among prison officers in Japan. Osaka City Med J 59, $91-8$.

18) Iwasaki S, Deguchi Y, Inoue K (2014) Effects of "Shinkeishitsu" on Occupational Stress in Japanese Workers. SOJ Psychology 1, 1-7.

19) Monsvold T, Bendixen M, Hagen R, Helvik AS (2011) Exposure to teacher bullying in schools: a study of patients with personality disorders. Nord J Psychiatry 65, 323-9.

20) Zung WW (1973) The differentiation of anxiety and depressive disorders: a psychopharmacological approach. Psychosomatics 14, 362-6.

21) Zung WW, Magruder-Habib K, Velez R, Alling W (1990) The comorbidity of anxiety and depression in general medical patients: a longitudinal study. The Journal of clinical psychiatry 51 Suppl, 77-80, discussion 81 .

22) Chida F, Okayama A, Nishi N, Sakai A (2004) Factor analysis of Zung Scale scores in a Japanese general population. Psychiatry Clin Neurosci 58, 420-6.

23) Kamibeppu K, Sugiura H, Nakajima K (2006) Factors affecting the consultation and actual condition of junior high school teachers who experienced depression counseling; in case of Tokyo prefecture. Mental Health Okamoto Memorial Foundation grants annual report 1, 43-50 (in Japanese).

24) Sakai K (2007) Fine teacher, Healthy child: Survey of teacher's health and recommendations. Science of labour 62, 325-32 (in Japanese).

25) Ministry of Health, Labour and Welfare. Provisional Report of Monthly Labour Survey-June 2015-. http://www. mhlw.go.jp/english/database/db-1/27/2706pe/2706pe.html. Accessed Jan 13, 2016.

26) Kitazoe N, lnoue S (2009) Mental Health among Inexperienced Teachers - Relation to the Job related Stress and tihe Coping behavior - . JPN Bull Soc Psychiat 18, 178-85 (in Japanese).

27) Kanchika M, Iwasaki S, Konish A, Deguchi Y, Kobayashi Y, Nakada A, Inoue K (2015) Aggression in Teachers is Related to Role Conflict and Role Ambiguity as Occupational Stress. Osaka City Med J 61, 93 - 104.

28) Ito M (2001) Teachers' burnout, and the association with the surroundings of school and individual factor. Health and Culture, Research-aid paper of the Yasuda Life Welfare Foundation 7, 8-14 (in Japanese).

29) Smith KJ (2010) A structural model of teacher role stress, satisfaction, commitment, and intentions to leave: a comment on Conley and You (2009). Psychol Rep 106, 576-8.

30) Mayers JK (1980) Use of self-report symptom scale to detect depression in a community sample. 137, 1081-4. 